\title{
Europäischer Tarifbericht des WSI 2010/2011
}

Thorsten Schulten

\begin{abstract}
Während die Konjunktur in den verschiedenen europäischen Ländern derzeit einen sehr unterschiedlichen Verlauf nimmt, fällt die Lohnentwicklung in Europa insgesamt äußerst schwach aus. Im Jahr 2010 kam es EU-weit zu einer Stagnation der Reallöhne. Für 2011 muss sogar mit einem deutlichen Absinken des Reallohnniveaus gerechnet werden. Verantwortlich hierfür ist nicht zuletzt die in der Europäischen Union vorherrschende Politik, die Lasten der Krise einseitig den Beschäftigten aufzubürden und Lohnkürzungen als vermeintliche Krisenlösungsstrategie zu propagieren. Damit ist die Lohnpolitik nach wie vor weit davon entfernt, einen Beitrag zur Überwindung der strukturellen Ungleichgewichte in Europa zu leisten und eine nachhaltige gesamteuropäische Wachstumsstrategie zu befördern.
\end{abstract}

\section{Ökonomische Rahmenbe- dingungen der Tarifpolitik}

\subsection{ALLGEMEINE WIRTSCHAFTSENT- WICKLUNG}

Nachdem die Tarifauseinandersetzungen im Jahr 2009 ganz im Zeichen der schwersten und tiefsten Wirtschaftskrise in der Geschichte der Europäischen Union (EU) gestanden hatten, hat sich die Konjunktur 2010 wieder deutlich erholt. Das Bruttoinlandsprodukt (BIP) stieg in diesem Jahr in der gesamten EU im Durchschnitt um 1,8 \%. Im Vorjahr war die Wirtschaftsleistung noch um 4,2 \% zurückgegangen. ${ }^{1}$ Allerdings lässt sich 2010 in Europa insgesamt eine Dreiteilung in der allgemeinen Konjunkturentwicklung beobachten (vgl. auch IMK et al. 2011): Erstens gibt es eine Ländergruppe, zu der insbesondere die stark exportorientierten Staaten wie Deutschland, Finnland, Schweden und die Slowakei gehören, die mit hohen Wachstumsraten von 3 bis 5,5\% gut aus der Krise herausgekommen ist. Im Gegensatz dazu hat sich die Krise insbesondere in den südeuropäischen Staaten wie Griechenland, Spanien, Portugal und in einigen osteuropäischen Ländern wie Bulgarien, Lettland und Rumänien sowie in Irland auch 2010 weiter fortgesetzt. Das am stärksten betroffene Land ist hierbei Griechenland, wo die Wirtschaftsleistung 2010 noch einmal um 4,5 \% zurückging. Schließlich gibt es eine dritte Gruppe von Staaten, zu der z.B. Frankreich und Großbritannien gehören, in der die konjunkturelle Erholung mit Wachstumsraten zwischen 1 und $2 \%$ eher verhalten ausfiel.

Nach den Prognosen der Europäischen Union wird sich diese Dreiteilung der eu- ropäischen Konjunkturentwicklung auch 2011 fortsetzen (Europäische Kommission 2011). Während sich die Wachstumsraten in den exportorientierten Volkswirtschaften gegenüber 2010 zwar etwas abschwächen, aber nach wie vor auf vergleichsweise hohem Niveau befinden werden, wird insbesondere für einige osteuropäische Staaten ein deutlich höheres Wachstum erwartet. Demgegenüber bleibt die ökonomische Lage in den südeuropäischen Ländern weiter prekär. Insgesamt erwartet die Europäische Kommission für 2011 ein EU-weites Wachstum von 1,8\%. Angesichts der anhaltenden Verschuldungskrise in vielen europäischen Staaten sowie der aufgrund mangelnder Regulierung nach wie vor fortbestehenden Krisenpotenziale auf den internationalen Finanzmärkten ist diese Prognose jedoch mit hohen Unsicherheiten behaftet.

\subsection{DIE LAGE AUF DEM ARBEITSMARKT}

Der unterschiedliche Konjunkturverlauf in Europa spiegelt sich auch in der Entwicklung auf dem Arbeitsmarkt wider (Tabelle 1). In vielen Ländern Süd- und Osteuropas sowie in Irland hat sich die im Krisenjahr 2009 stark angestiegene Arbeitslosigkeit 2010 noch einmal erhöht. In zehn von 27 EU-Staaten betrug die Arbeitslosenquote mehr als $10 \%$. Die EU-weit höchste Arbeitslosenquote gab es in Spanien mit $20 \%$, gefolgt von den baltischen Staaten Lettland und Litauen mit 18,7 bzw. $17,8 \%$. In weiteren 14 EU-Staaten bewegte sich die Arbeitslosenquote zwischen 6 und $10 \%$, wobei die meisten Länder 2010 nochmals einen leichten Anstieg verzeichneten. Lediglich in drei EU-Staaten (Luxemburg, Niederlande und Österreich) lag die Arbeitslosenquote unter $5 \%$. Die geringste
Arbeitslosenquote gab es hingegen außerhalb der EU in Norwegen mit 3,5\% und der Schweiz mit 2,8\%. Zusammen mit Luxemburg war Deutschland das einzige Land in der EU, in dem die Arbeitslosigkeit 2010 wieder zurückgegangen ist und dabei sogar das Vorkrisenniveau des Jahres 2008 unterschritten hat.

Im Jahresdurchschnitt 2010 waren EU-weit mehr als 23 Mio. Menschen offiziell arbeitslos, was einer Arbeitslosenquote von 9,6\% entspricht. Für 2011 erwartet die Europäische Kommission im EU-Durchschnitt kaum Veränderungen auf dem Arbeitsmarkt. In einigen Krisenländern wird die Arbeitslosigkeit weiter ansteigen, während einige wenige Länder - darunter insbesondere Deutschland und Schweden - ihre Arbeitslosenquoten weiter verringern können. Angesichts der anhaltend hohen Arbeitslosigkeit bleibt die Verhandlungsmacht der Gewerkschaften in den meisten europäischen Ländern damit weiterhin stark beschränkt, was sich deutlich dämpfend auf die Lohnentwicklung auswirkt.

\footnotetext{
Im Folgenden wird, falls nicht anders ausgewiesen auf Daten der Annual Macro-Economic Database (AMECO) zurückgegriffen, die von der Generaldirektion Wirtschaft und Finanzen (GD ECFIN) der Europäischen Kommission bereitgestellt wird (http://ec.europa.eu/economy_finance/db_ indicators/ameco/index_en.htm). Bei den Angaben für 2010 handelt es sich teilweise noch um vorläufige Daten. Bei den Angaben für 2011 handelt es sich um Prognosedaten der Europäischen Kommission (vgl. Europäische Kommission 2011)
}

Thorsten Schulten, Dr., Wissenschaftler im Wirtschafts- und Sozialwissenschaftliches Institut (WSI) in der Hans-Böckler-Stiftung. Arbeitsschwerpunkte: Arbeits- und Tarifpolitik in Europa. e-mail: thorsten-schulten@boeckler.de 


\subsection{PREIS- UND PRODUKTIVITÄTS- ENTWICKLUNG}

Die Tarifauseinandersetzungen in Europa orientieren sich traditionell neben der Konjunkturentwicklung und der Lage auf dem Arbeitsmarkt vor allem an der Entwicklung der Preise und der Arbeitsproduktivität (Tabelle 2). Die Summe beider Indikatoren bildet den lohnpolitischen Verteilungsspielraum, dessen Ausschöpfung durch entsprechende Lohnerhöhungen den Beschäftigten eine gleichgewichtige Partizipation an der allgemeinen Wirtschaftentwicklung garantiert und das Verhältnis zwischen Arbeits- und Kapitaleinkommen konstant hält. Bei der Definition des lohnpolitischen Verteilungsspielraumes als Summe aus Preis- und Produktivitätsentwicklung ist in der Wirtschaftswissenschaft strittig, welche konkreten Indikatoren insbesondere für die Preisentwicklung verwendet werden sollen. Gegenüber möglichen alternativen Indikatoren (wie z.B. der Kerninflationsrate, der Zielinflationsrate der Europäischen Zentralbank (EZB) oder der Entwicklung der Erzeugerpreise) wird im Folgenden bei der Bestimmung der nationalen Verteilungsspielräume die jeweils nationale Entwicklung der Verbraucherpreise zugrunde gelegt. Aus Arbeitnehmersicht stellen diese den wichtigsten ökonomischen Indikator dar, weil sie über das reale Lohnniveau und die damit verbundene Kaufkraft entscheiden. Dementsprechend bilden die nationalen Verbraucherpreise für die Gewerkschaften die bedeutsamste Orientierungsgröße bei den Tarifauseinandersetzungen.

Nachdem im Krisenjahr 2009 die Entwicklung des harmonisierten Verbraucherpreisindex (HVPI) EU-weit mit 1,0\% äußerst gering ausfiel und in einigen europäischen Ländern sogar deutlich deflationäre Züge aufwies, hat sie 2010 mit 2,1\% wieder ein Niveau erreicht, das in etwa der Zielinflationsrate der EZB entspricht ( $\mathrm{Ta}$ belle 2). Den höchsten Preisauftrieb gab es mit 6,1 \% in Rumänien, gefolgt von jeweils $4,7 \%$ in Griechenland und Ungarn und 3,3\% in Großbritannien. Demgegenüber waren Irland und Lettland die einzigen beiden Staaten mit einer nach wie vor negativen Preisentwicklung. Für 2011 erwartet die Europäische Kommission einen deutlichen Anstieg der Verbraucherpreise auf durchschnittlich 3,0 \%, was vor allem auf die Entwicklung der internationalen Rohstoff- und Energiepreise zurückgeführt wird.

Tabelle 1: Wachstum und Arbeitslosigkeit in Europa 2008-2011*

\begin{tabular}{|c|c|c|c|c|c|c|c|c|}
\hline & \multicolumn{4}{|c|}{ Bruttoinlandsprodukt } & \multicolumn{4}{|c|}{ Arbeitslosigkeit } \\
\hline & 2008 & 2009 & 2010 & 2011 & 2008 & 2009 & 2010 & 2011 \\
\hline \multicolumn{9}{|l|}{ Alte EU-Staaten } \\
\hline Belgien & 1,0 & $-2,8$ & 2,2 & 2,4 & 7,0 & 7,9 & 8,3 & 7,9 \\
\hline Dänemark & $-1,1$ & $-5,2$ & 2,1 & 1,7 & 3,3 & 6,0 & 7,4 & 7,1 \\
\hline Deutschland & 1,0 & $-4,7$ & 3,6 & 2,6 & 7,5 & 7,8 & 7,1 & 6,4 \\
\hline Finnland & 0,9 & $-8,2$ & 3,1 & 3,7 & 6,4 & 8,2 & 8,4 & 7,9 \\
\hline Frankreich & 0,2 & $-2,6$ & 1,6 & 1,8 & 7,8 & 9,5 & 9,7 & 9,5 \\
\hline Griechenland & 1,0 & $-2,0$ & $-4,5$ & $-3,5$ & 7,7 & 9,5 & 12,6 & 15,2 \\
\hline Großbritannien & $-0,1$ & $-4,9$ & 1,3 & 1,7 & 5,6 & 7,6 & 7,8 & 8,0 \\
\hline Irland & $-3,5$ & $-7,6$ & $-1,0$ & 0,6 & 6,3 & 11,9 & 13,7 & 14,6 \\
\hline Italien & $-1,3$ & $-5,2$ & 1,3 & 1,0 & 6,7 & 7,8 & 8,4 & 8,4 \\
\hline Luxemburg & 1,4 & $-3,6$ & 3,5 & 3,4 & 4,9 & 5,1 & 4,5 & 4,4 \\
\hline Niederlande & 1,9 & $-3,9$ & 1,8 & 1,9 & 3,1 & 3,7 & 4,5 & 4,2 \\
\hline Österreich & 2,2 & $-3,9$ & 2,0 & 2,4 & 3,8 & 4,8 & 4,4 & 4,3 \\
\hline Portugal & 0,0 & $-2,5$ & 1,3 & $-2,2$ & 7,7 & 9,6 & 11,0 & 12,3 \\
\hline Schweden & $-0,6$ & $-5,3$ & 5,5 & 4,2 & 6,2 & 8,3 & 8,4 & 7,6 \\
\hline Spanien & 0,9 & $-3,7$ & $-0,1$ & 0,8 & 11,3 & 18,0 & 20,1 & 20,6 \\
\hline \multicolumn{9}{|l|}{ Neue EU-Staaten } \\
\hline Bulgarien & 6,2 & $-5,5$ & 0,2 & 2,8 & 5,6 & 6,8 & 10,2 & 9,4 \\
\hline Estland & $-5,1$ & $-13,9$ & 3,1 & 4,9 & 5,5 & 13,8 & 16,9 & 13,0 \\
\hline Lettland & $-4,2$ & $-18,0$ & $-0,3$ & 3,3 & 7,5 & 17,1 & 18,7 & 17,2 \\
\hline Litauen & 2,9 & $-14,7$ & 1,3 & 5,0 & 5,8 & 13,7 & 17,8 & 15,5 \\
\hline Malta & 5,3 & $-3,4$ & 3,7 & 2,0 & 5,9 & 7,0 & 6,8 & 6,8 \\
\hline Polen & 5,1 & 1,7 & 3,8 & 4,0 & 7,1 & 8,2 & 9,6 & 9,3 \\
\hline Rumänien & 7,3 & $-7,1$ & $-1,3$ & 1,5 & 5,8 & 6,9 & 7,3 & 7,2 \\
\hline Slowakei & 5,8 & $-4,8$ & 4,0 & 3,5 & 9,5 & 12,0 & 14,4 & 14,0 \\
\hline Slowenien & 3,7 & $-8,1$ & 1,2 & 1,9 & 4,4 & 5,9 & 7,3 & 8,2 \\
\hline Ungarn & 0,8 & $-6,7$ & 1,2 & 2,7 & 7,8 & 10,0 & 11,2 & 11,0 \\
\hline Tschechien & 2,5 & $-4,1$ & 2,3 & 2,0 & 4,4 & 6,7 & 7,3 & 6,8 \\
\hline Zypern & 3,6 & $-1,7$ & 1,0 & 1,5 & 3,6 & 5,3 & 6,5 & 6,3 \\
\hline Gesamte EU 27 & 0,5 & $-4,2$ & 1,8 & 1,8 & 7,1 & 9,0 & 9,6 & 9,5 \\
\hline Norwegen & 0,8 & $-1,4$ & 0,4 & 2,7 & 2,5 & 3,1 & 3,5 & 3,5 \\
\hline Schweiz & 1,9 & $-1,9$ & 2,6 & 1,9 & 3,2 & 2,9 & 2,8 & 2,8 \\
\hline \multicolumn{9}{|c|}{$\begin{array}{l}\text { *Bruttoinlandsprodukt }=\text { Veränderung des BIP in \% gegenüber dem Vorjahr. } \\
\text { Arbeitslosenquote }=\text { Anzahl der Arbeitslosen in \% der zivilen Erwerbsbevölkerung } \\
\text { (Eurostat-Definition). } \\
\text { Angaben für 2011: Prognose der Europäischen Kommission. }\end{array}$} \\
\hline Quelle: AMECO Da & Versior & 3.5.2011 & & & & & & \\
\hline
\end{tabular}

Die Arbeitsproduktivität hat $2010 \mathrm{mit}$ einem Zuwachs von EU-weit 2,3 \% wieder merklich zugelegt (Tabelle 2). Im Vorjahr war sie angesichts der Krise um 2,3 \% zurückgegangen. Die höchsten Produktivitätszuwächse konnten einige osteuropäische Staaten verzeichnen, die im Jahr zuvor einen besonders starken Produktivitätseinbruch erlebten. In Westeuropa kam es vor allem in den exportorientierten Volkswirtschaften zu einem überdurchschnittlichen Produktivitätsanstieg, der ebenfalls teilweise auf das Auslaufen produktivitätssenkender Kriseninterventionsmaßnahmen (wie z.B. Kurzarbeit) zurückzuführen ist. Einmal mehr wird die besondere Lage Griechenlands unterstrichen, das als einziges EU-Land 2010 einen Produktivitätsrückgang erlebte. Nach den Prognosen der Europäischen Kommission werden sich die Produktivitätszuwächse im Jahr 2011 auf durchschnittlich 1,4\% wieder deutlich verlangsamen, wobei neben Griechenland auch für Portugal ein weiterer Rückgang der Produktivität vorhergesagt wird.

Aufgrund der gestiegenen Preis- und Produktivitätsentwicklung ergab sich im Jahr 2010 im EU-Durchschnitt ein lohnpolitischer Verteilungsspielraum von 4,3\% (Tabelle 2). Der rechnerisch größte Verteilungsspielraum existierte mit $11 \%$ in Lettland, gefolgt von einer Reihe weiterer osteuropäischer Länder mit Werten zwischen 6 und $10 \%$. Diese Länder haben damit die ökonomischen Voraussetzungen geschaffen, den lohnpolitischen Aufholprozess gegenüber Westeuropa wieder aufzunehmen, der zuvor durch die Krise unterbrochen bzw. teilweise sogar rückgängig gemacht wurde. In Westeuropa lag der lohnpolitische Verteilungsspielraum 2010 in den meisten Ländern zwischen 3 und $5 \%$. Die höchsten Werte gab es mit 6,4 bzw. 6,3 \% in Dänemark und Schweden, während Griechenland und Irland mit 2,3 bzw. 1,7 \% die niedrigsten Werte aufwiesen. 
Tabelle 2: Preise, Arbeitsproduktivität und Verteilungsspielraum in Europa 2008-2011*

\begin{tabular}{|c|c|c|c|c|c|c|c|c|c|c|c|c|}
\hline & \multicolumn{4}{|c|}{ Preise } & \multicolumn{4}{|c|}{ Arbeitsproduktivität } & \multicolumn{4}{|c|}{ Verteilungsspielraum } \\
\hline & 2008 & 2009 & 2010 & 2011 & 2008 & 2009 & 2010 & 2011 & 2008 & 2009 & 2010 & 2011 \\
\hline \multicolumn{13}{|l|}{ Alte EU-Staaten } \\
\hline Belgien & 4,5 & $-0,0$ & 2,3 & 3,6 & $-0,7$ & $-2,4$ & 1,5 & 1,6 & 3,8 & $-2,4$ & 3,8 & 5,1 \\
\hline Dänemark & 3,6 & 1,1 & 2,2 & 2,5 & $-2,9$ & $-2,2$ & 4,2 & 1,6 & 0,7 & $-1,1$ & 6,4 & 4,1 \\
\hline Deutschland & 2,8 & 0,2 & 1,2 & 2,6 & $-0,4$ & $-4,7$ & 3,1 & 1,6 & 2,4 & $-4,5$ & 4,2 & 4,2 \\
\hline Finnland & 3,9 & 1,6 & 1,7 & 3,6 & $-0,6$ & $-5,6$ & 3,5 & 2,7 & 3,3 & $-4,0$ & 5,2 & 6,3 \\
\hline Frankreich & 3,2 & 0,1 & 1,7 & 2,2 & $-0,5$ & $-1,4$ & 1,5 & 0,9 & 2,7 & $-1,3$ & 3,2 & 3,2 \\
\hline Griechenland & 4,2 & 1,3 & 4,7 & 2,4 & 0,8 & $-1,3$ & $-2,4$ & $-0,9$ & 5,1 & 0,0 & 2,3 & 1,5 \\
\hline Großbritannien & 3,6 & 2,2 & 3,3 & 4,1 & $-0,8$ & $-3,4$ & 1,0 & 1,3 & 2,8 & $-1,2$ & 4,3 & 5,4 \\
\hline Irland & 3,1 & $-1,7$ & $-1,6$ & 1,0 & $-2,4$ & 0,6 & 3,2 & 2,2 & 0,7 & $-1,1$ & 1,7 & 3,2 \\
\hline Italien & 3,5 & 0,8 & 1,6 & 2,6 & $-0,9$ & $-2,6$ & 2,0 & 0,6 & 2,6 & $-1,9$ & 3,7 & 3,1 \\
\hline Luxemburg & 4,1 & 0,0 & 2,8 & 3,5 & $-3,2$ & $-4,5$ & 1,9 & 1,3 & 0,9 & $-4,5$ & 4,7 & 4,8 \\
\hline Niederlande & 2,2 & 1,0 & 0,9 & 2,2 & 0,6 & $-2,8$ & 2,4 & 1,4 & 2,9 & $-1,8$ & 3,3 & 3,7 \\
\hline Österreich & 3,2 & 0,4 & 1,7 & 2,9 & 0,5 & $-2,3$ & 1,0 & 1,6 & 3,8 & $-1,9$ & 2,7 & 4,4 \\
\hline Portugal & 2,7 & $-0,9$ & 1,4 & 3,4 & $-0,5$ & $-0,0$ & 2,9 & $-0,8$ & 2,2 & $-0,9$ & 4,3 & 2,6 \\
\hline Schweden & 3,3 & 1,9 & 1,9 & 1,7 & $-1,5$ & $-3,4$ & 4,4 & 2,2 & 1,8 & $-1,4$ & 6,3 & 3,9 \\
\hline Spanien & 4,1 & $-0,2$ & 2,0 & 3,0 & 1,4 & 3,1 & 2,3 & 1,4 & 5,5 & 2,9 & 4,3 & 4,4 \\
\hline \multicolumn{13}{|l|}{ Neue EU-Staaten } \\
\hline Bulgarien & 12,0 & 2,5 & 3,0 & 4,3 & 3,5 & $-2,9$ & 6,4 & 2,3 & 15,4 & $-0,5$ & 9,4 & 6,6 \\
\hline Estland & 10,6 & 0,2 & 2,7 & 4,7 & $-5,2$ & $-4,4$ & 8,3 & 0,6 & 5,4 & $-4,2$ & 11,1 & 5,3 \\
\hline Lettland & 15,3 & 3,3 & $-1,2$ & 3,4 & $-5,1$ & $-5,5$ & 4,6 & 1,8 & 10,1 & $-2,3$ & 3,4 & 5,2 \\
\hline Litauen & 11,1 & 4,2 & 1,2 & 3,2 & 3,6 & $-8,5$ & 6,8 & 2,8 & 14,7 & $-4,3$ & 8,0 & 6,0 \\
\hline Malta & 4,7 & 1,8 & 2,0 & 2,7 & 2,6 & $-3,1$ & 1,4 & 0,7 & 7,3 & $-1,2$ & 3,5 & 3,4 \\
\hline Polen & 4,2 & 4,0 & 2,7 & 3,8 & 1,3 & 1,3 & 3,4 & 2,9 & 5,5 & 5,3 & 6,1 & 6,7 \\
\hline Rumänien & 7,9 & 5,6 & 6,1 & 6,7 & 7,3 & $-5,4$ & 0,5 & 1,3 & 15,2 & 0,2 & 6,6 & 8,0 \\
\hline Slowakei & 3,9 & 0,9 & 0,7 & 3,6 & 2,8 & $-2,3$ & 5,5 & 2,9 & 6,7 & $-1,4$ & 6,2 & 6,6 \\
\hline Slowenien & 5,5 & 0,9 & 2,1 & 2,6 & 0,9 & $-6,4$ & 3,4 & 3,3 & 6,5 & $-5,5$ & 5,5 & 5,8 \\
\hline Tschechien & 6,3 & 0,6 & 1,2 & 2,3 & 1,2 & $-3,0$ & 3,1 & 2,0 & 7,5 & $-2,4$ & 4,4 & 4,3 \\
\hline Ungarn & 6,0 & 4,0 & 4,7 & 4,0 & 2,1 & $-4,0$ & 1,0 & 2,3 & 8,2 & 0,1 & 5,7 & 6,3 \\
\hline Zypern & 4,4 & 0,2 & 2,6 & 3,4 & 0,8 & $-1,0$ & 1,3 & 1,3 & 5,2 & $-0,8$ & 3,9 & 4,7 \\
\hline Gesamte EU 27 & 3,7 & 1,0 & 2,1 & 3,0 & 0,3 & $-2,3$ & 2,3 & 1,4 & 3,9 & $-1,3$ & 4,3 & 4,4 \\
\hline Norwegen & 3,4 & 2,3 & 2,3 & 1,9 & $-2,2$ & $-0,8$ & 0,6 & 2,2 & 1,2 & 1,5 & 3,0 & 4,0 \\
\hline Schweiz & 2,3 & $-0,7$ & 0,6 & 1,0 & 0,0 & $-4,1$ & 0,4 & $-0,3$ & 2,4 & $-4,7$ & 1,0 & 0,7 \\
\hline $\begin{array}{l}\text { *Preise }=\text { Harmonisi } \\
\text { Arbeitsproduktivität } \\
\text { Verteilungsspielraun } \\
\text { Angaben für 2011: }\end{array}$ & $\begin{array}{l}\text { aucherpre } \\
\text { IP pro Ery } \\
\text { aus Preis } \\
\text { er Europäi }\end{array}$ & $\begin{array}{l}\text { dex (HVP } \\
\text { ostätigen, } \\
\text { d Arbeits } \\
\text { en Komm }\end{array}$ & $\begin{array}{l}\text { Veränder } \\
\text { ränderun } \\
\text { duktivitä } \\
\text { on. }\end{array}$ & $\begin{array}{l}\text { in \% geg } \\
\% \text { gegen } \\
\text { twicklung }\end{array}$ & Vorjahr. & & & & & & & \\
\hline Quelle: AMECO Da & er Europä & en Komm & on, Versic & 13.5.2011 & ungen $\mathrm{d}$ & & & & & & & \\
\hline
\end{tabular}

Im Jahr 2011 wird der lohnpolitische Verteilungsspielraum durch die rückläufige Produktivitätsentwicklung zwar geschmälert. Dies wird durch die höhere Preisentwicklung jedoch wieder ausgeglichen, sodass sich insgesamt mit $4,4 \%$ ein lohnpolitischer Verteilungsspielraums ergibt, der etwa auf dem Niveau des Vorjahres liegt. Orientiert man sich bei der Berechnung des Verteilungsspielraums nicht an der jeweils jahresbezogenen Produktivität, sondern an der sogenannten Trendproduktivität, die sich z.B. aus der durchschnittlichen Produktivitätsentwicklung der letzten zehn Jahre ergibt, so besteht derzeit im EU-Durchschnitt ein Verteilungsrahmen für nominale Lohnerhöhungen von 3,5 bis $4 \%$. Eine solche mittelfristige Orientierung kann zu einer Verstetigung der Lohnpolitik beitragen und zugleich verhindern, das in Ausnahmesituationen wie dem Krisenjahr 2009, in dem fast alle europäischen Länder einen negativen Verteilungsspielraum ver- zeichneten, die Lohnpolitik pro-zyklisch agiert und durch mögliche Lohnkürzungen die Krise weiter verschärft.

\section{Die Entwicklung der
Tariflöhne}

Die Analyse der Tariflohnentwicklung in Europa steht vor dem grundsätzlichen Problem, dass es bis heute keine offizielle europäische Tariflohnstatistik mit harmonisierten Tarifdaten gibt. ${ }^{2}$ Der einzige bislang zur Verfügung stehende offizielle Indikator ist der Tariflohnindex für die Euro-Zone, der von der EZB berechnet wird. Die Grundlage hierfür bilden nichtharmonisierte nationale Tarifdaten aus neun Mitgliedstaaten der Europäischen Währungsunion (EWU), die insgesamt etwa $95 \%$ des Euro-Raums abdecken.
Hierzu gehören die großen EWU-Länder Deutschland, Frankreich, Italien und Spanien sowie Belgien, Finnland, Österreich,

2 Seit einigen Jahren veröffentlicht das European Industrial Relations Observatory (EIRO) regelmäßig Daten über die Entwicklung der Tariflöhne in Europa (vgl. zuletzt Carley 2010). Eine Überprüfung der vom EIRO verwendeten nationalen Ursprungsdaten macht jedoch deutlich, dass diese große methodische Mängel aufweisen und nicht miteinander vergleichbar sind. Zum einen werden in den EIRO-Daten mitunter sehr unterschiedliche Bereiche und Zeiträume erfasst. Zum anderen werden aufgrund fehlender nationaler Tariflohnstatistiken oft Datenquellen verwendet, die anstatt der Tariflohnentwicklungen die Effektivlohnentwicklungen darstellen. Qualitative Berichte über die Tarifauseinandersetzungen in den einzelnen EU-Staaten finden sich in den laufenden nationalen Beiträgen zum EIRO (http://www.eurofound. europa.eu/eiro) sowie in dem gemeinsam vom Europäischen Gewerkschaftsinstitut und dem Amsterdam Institute for Advanced Labour Studies (AIAS) herausgegebenen Collective Bargaining Newsletter (http://www.etui.org/research/Publications/ Newsletters/Collective-bargaining-newsletter). 


\begin{tabular}{|c|c|c|c|}
\hline Land & Bezeichnung & Quelle & Internet \\
\hline Belgien & Index van de conventionele lonen, Arbeiders & Federale Overheidsdienst & http://www.werk.belgie.be \\
\hline \multirow[t]{2}{*}{ Deutschland } & Tariflöhne & WSI-Tarifarchiv & http://www.tarifvertrag.de \\
\hline & Tariflöhne (Monatsbasis) & Bundesbank & http://www.bundesbank.de \\
\hline Finnland & Index of wage and salary earnings & Statistics Finland & http://www.stat.fi \\
\hline Frankreich & Salaire mensuel de base de l'ensemble des salariés & DARES & http://www.travail-solidarite.gouv.fr \\
\hline Italien & Retribuzioni contrattuali per dipendente & Istat & http://www.istat.it/ \\
\hline Niederlande & Cao-lonen per maand incl.bijz.beloningen & Centraal Bureau voor de Statistiek & http://statline.cbs.nl \\
\hline Österreich & Tariflohnindex & Statistik Austria & http://www.statistik.at \\
\hline Portugal & Wages: Collective agreements & Banco de Portugal & http://www.bportugal.pt \\
\hline Spanien & Aumento Salarial Convenios Colectivos de Trabajo & Ministerio de Trabajo & http://www.mtin.es/ \\
\hline Euro-Zone & Negotiated Wages & EZB & http://www.ecb.int \\
\hline Schweden & Short Term Wage and Salary Statistics & National Mediation Office & http://www.mi.se \\
\hline Schweiz & Löhne nach Gesamtarbeitsverträgen & Bundesamt für Statistik & http://www.bfs.admin.ch \\
\hline
\end{tabular}

Quelle: Zusammenstellung des WSI.

WSI MITTEILUNGEN

die Niederlande und Portugal, deren Tarifdaten durch die Arbeitsministerien, die nationalen Statistikämter oder die nationalen Zentralbanken erhoben werden. (Übersicht 1).

Während die EZB lediglich den aggregierten Tariflohnindex für die gesamte Euro-Zone publiziert, werden hier auch die hierbei zugrunde liegenden nationalen Tarifdaten veröffentlicht. Für Deutschland werden von der EZB die Daten des Tariflohnindex der Deutschen Bundesbank verwendet. Diese unterscheiden sich zum Teil deutlich von den Daten, die das Tarifarchiv des Wirtschafts- und Sozialwissenschaftlichen Instituts (WSI-Tarifarchiv) in der Hans-Böckler-Stiftung jeweils für die kalenderjährliche Tariflohnerhöhung berechnet. ${ }^{3}$ Im Folgenden werden deshalb für Deutschland sowohl die Daten der Bundesbank als auch des WSI-Tarifarchivs dargestellt. Außerdem wurde der EZBTariflohnindex für die Euro-Zone auf der Grundlage der WSI-Tarifarchiv-Daten für Deutschland neu kalkuliert und er wird hier ebenfalls doppelt ausgewiesen. Neben den Ländern der Euro-Zone werden darüber hinaus auch die vorliegenden Tariflohndaten für Schweden und die Schweiz mit in die Analyse einbezogen. Für die übrigen europäischen Länder - darunter alle Staaten aus Mittel und Osteuropa - liegen zumeist keine Tariflohnstatistiken vor.

Im Jahr 2010 sind die nominalen Tariflöhne in der Euro-Zone um durchschnittlich 1,7\% angestiegen (Abbildung 1). Die Spannbreite reichte von Finnland mit einer nominalen Tariflohnerhöhung von $2,6 \%$ bis zu Belgien mit 0,7\%. Deutschland bewegte sich mit 1,6\% (Bundesbank) bzw. 1,8 \% (WSI) im europäischen
Abb. 1: Nominale Entwicklung der Tariflöhne 2009 und 2010 - in \% zum Vorjahr -

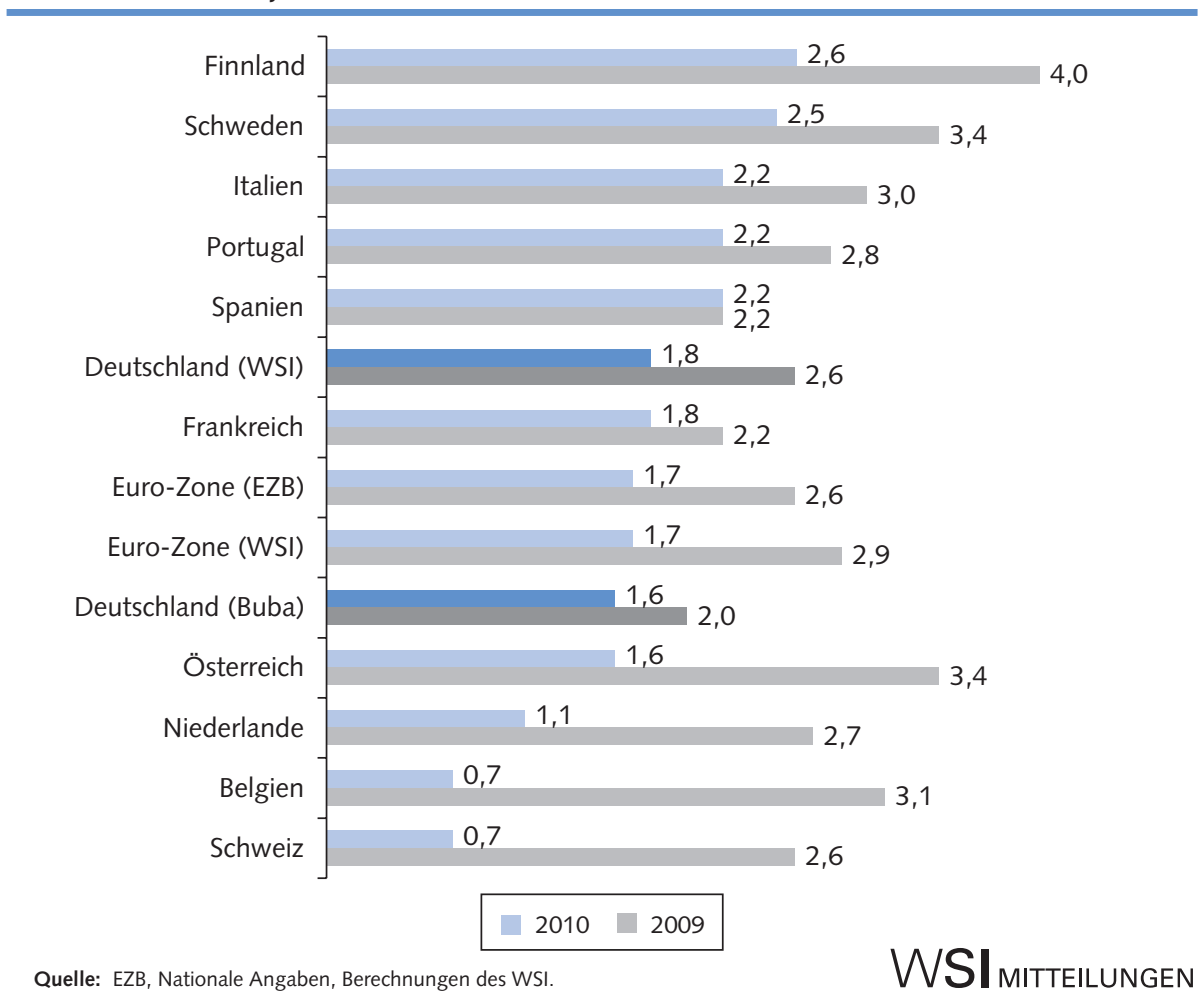

Durchschnitt. Gegenüber dem Vorjahr sind Tariflohnsteigerungen in den meisten Ländern deutlich geringer ausgefallen. So betrug der durchschnittliche Zuwachs der Tariflöhne in der Euro-Zone 2009 2,6 \% (EZB) bzw. 2,9 \% (WSI). Offensichtlich hat sich die Krise erst mit einer gewissen Zeitverzögerung auf die Tariflöhne ausgewirkt. Während im Jahr 2009 noch vielfach Tarifabschlüsse aus den Vorkrisenjahren 2008 und 2007 wirksam wurden, schlägt sich 2010 die Krise in deutlich niedrigeren Tariflohnsteigerungen nieder.
3 Zur Berechnungsmethode des WSI-Tarifarchivs vgl. den Beitrag von Reinhard Bispinck in diesem Heft. Ein wesentlicher Unterschied zu den Daten der Bundesbank liegt darin, dass das WSI lediglich diejenigen Tarifabschlüsse berücksichtigt, die in dem jeweiligen Kalenderjahr wirksam werden. Im Gegensatz dazu schreibt die Bundesbank einen festen Tariflohnindex fort, bei dem Branchen, die in einem Kalenderjahr auslaufen ohne im selben Jahr erneuert zu werden, mit einer Lohnsteigerung von $0 \%$ berücksichtigt werden. Dementsprechend liegen die Bundesbankzahlen in einigen Jahren deutlich unterhalb der Zahlen des WSI-Tarifarchivs. 
Abb. 2: Reale Entwicklung der Tariflöhne 2009 und 2010*

- in \% zum Vorjahr -

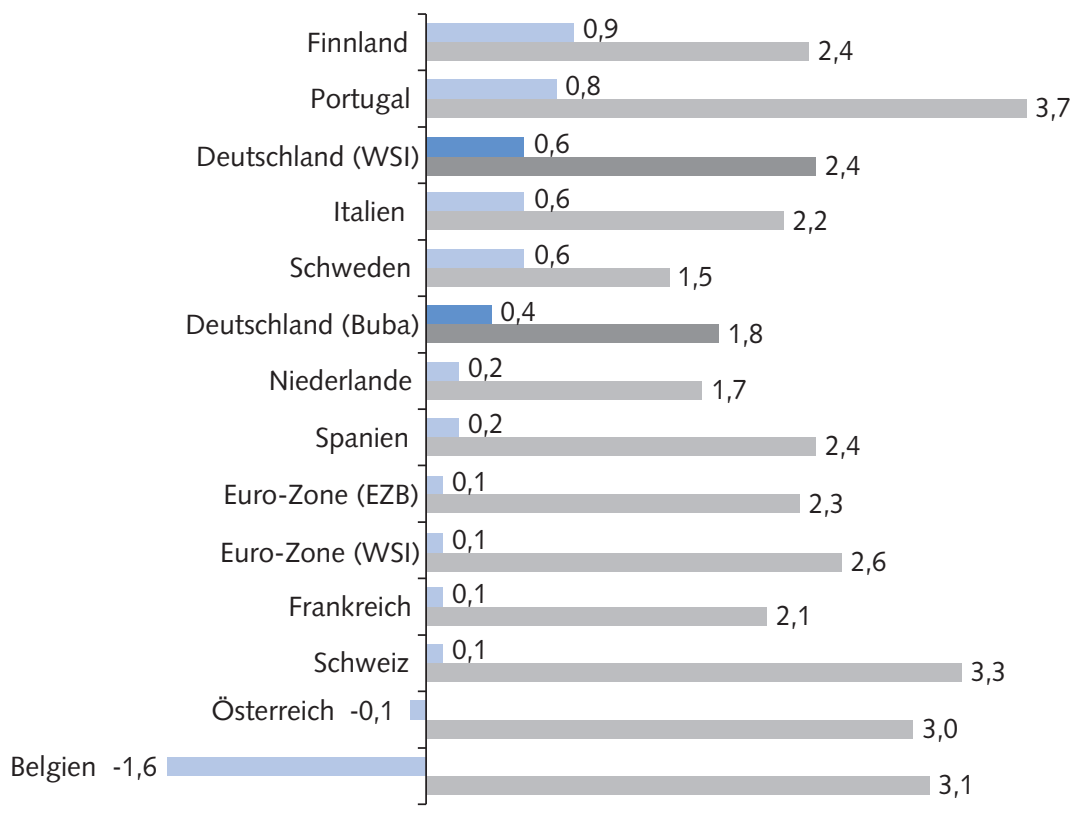

$2010 \square 2009$

*deflationiert um den Harmonisierten Verbraucherindex.

Quelle: EZB, Nationale Angaben, Berechnungen des WSI.

WSI MITTEILUNGEN

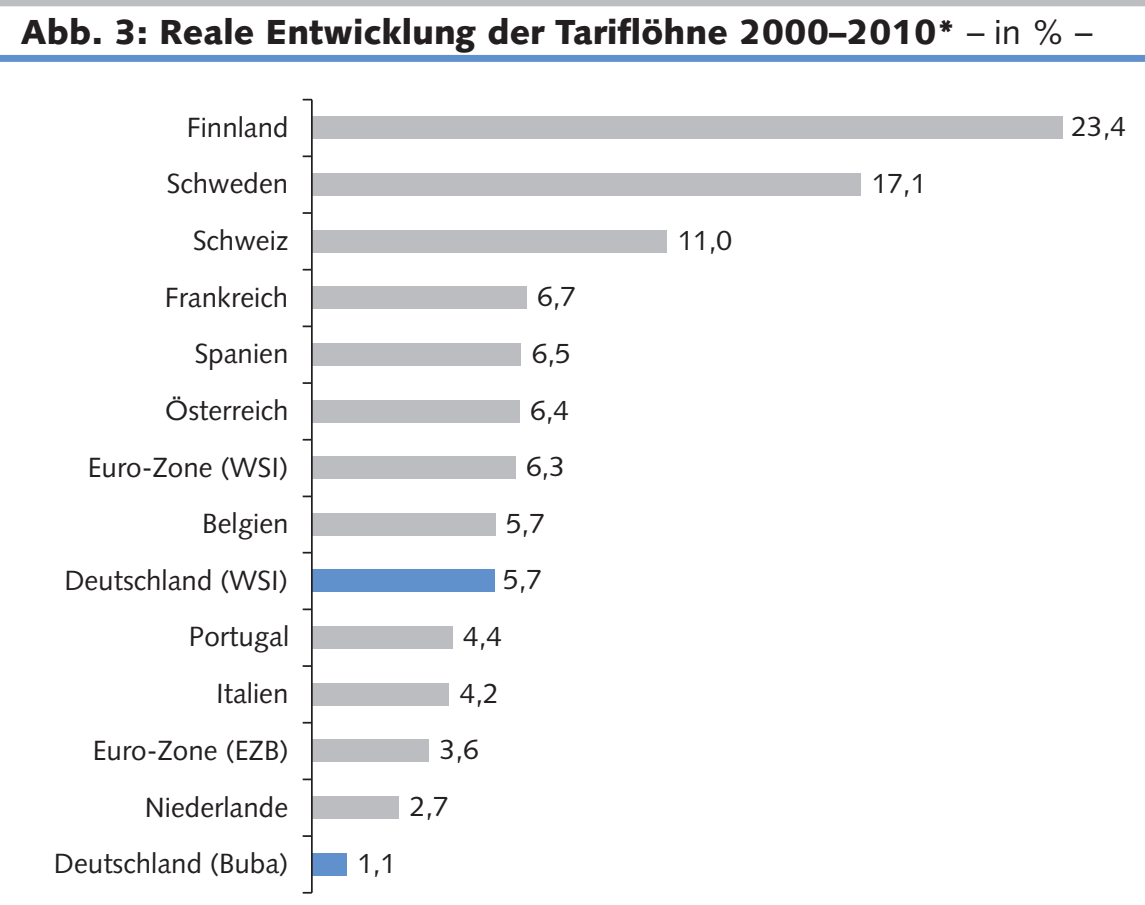

* deflationiert um den Harmonisierten Verbraucherpreisindex.

Quelle: EZB, Nationale Angaben, Berechnungen des WSI.

WSI MITTEILUNGEN

Bei der Betrachtung der um die Entwicklung der Verbraucherpreise bereinigten realen Tariflohnentwicklung wird deutlich, dass nach kräftigen Reallohnerhöhungen gen die realen Tariflöhne im Durchschnitt lediglich um 0,1 \% gegenüber 2,3\% (EZB) bzw. 2,6\% (WSI) im Vorjahr. Während Finnland mit einem Plus von $0,9 \%$ noch die höchsten realen Tariflohnsteigerungen verzeichnete, gingen die Tariflöhne in Belgien real sogar um 1,6 \% zurück. Aufgrund der in Belgien bestehenden automatischen Indexierung der Löhne an die Preisentwicklung dürfte dieser Realeinkommensverlust jedoch 2011 ausgeglichen werden. Deutschland bewegte sich mit einem realen Tarifplus von 0,4\% (Bundesbank) bzw. 0,6\% (WSI) im oberen Drittel.

Über das gesamte letzte Jahrzehnt hinweg zeigt die Entwicklung der Tariflöhne in Europa sehr große Unterschiede (Abbildung 3). Die größten realen Tariflohnsteigerungen gab es mit mehr als $23 \%$ in Finnland, gefolgt von Schweden mit gut $17 \%$ und der Schweiz mit $11 \%$. Für Deutschland variieren die Werte je nach Datenquelle zwischen einem realen Tarifplus von 5,7\% (WSI) und lediglich 1,1\% (Bundesbank). Nach den Bundesbankzahlen ist Deutschland auch bei den Tariflöhnen das absolute Schlusslicht in Europa, während es sich nach den Zahlen des WSI-Tarifarchivs im unteren Drittel befindet. In der Euro-Zone stiegen die realen Tariflöhne im letzten Jahrzehnt um 3,6 \% (EZB) bzw. 6,3\% (WSI).

\section{Die Entwicklung der Effektivlöhne}

Im Gegensatz zu den Tariflöhnen, die auf tarifvertraglichen Vereinbarungen beruhen, handelt es sich bei den Effektivlöhnen um die in der Volkswirtschaftlichen Gesamtrechnung (VGR) ausgewiesenen Löhne, die tatsächlich an alle Beschäftigte gezahlt wurden. Der oft zu beobachtende Unterschied zwischen Tarif- und Effektivlohnentwicklung wird als Lohndrift bezeichnet und kann verschiedene Ursachen haben. Inwieweit die Tariflohnerhöhungen tatsächlich auch effektiv beim Durchschnitt aller Beschäftigten ankommen, hängt vor allem von der Tarifbindung ab, die in Europa zwischen mehr als $90 \%$ in einigen westeuropäischen Ländern und weniger als $20 \%$ in den baltischen Staaten variiert (Schulten 2010). Darüber hinaus besteht in einigen Ländern die Möglichkeit, unter bestimmten Bedingungen auf betrieblicher 
Ebene von sektoralen Tariferhöhungen nach unten hin abzuweichen. Umgekehrt werden in vielen Ländern von den Unternehmen nach wie vor übertarifliche Leistungen bezahlt, die über den Tarifabschlüssen liegen. Schließlich wird das Verhältnis von Tarif- und Effektivlöhnen auch durch die Entwicklung der effektiven Arbeitszeit beeinflusst, wobei z.B. Überstunden eine positive, Kurzarbeit hingegen eine negative Lohndrift befördern. Im Folgenden wird die Entwicklung der Effektivlöhne auf der Basis der AMECO-Datenbank der Europäischen Kommission analysiert. ${ }^{4}$

Im Jahr 2010 stiegen die effektiven Nominallöhne im EU-Durchschnitt um 2,1 \% und damit etwas stärker als im Vorjahr, wo sie lediglich um 1,6\% zunahmen (Tabelle 3). Die höchsten Nominallohnzuwachsraten gab es in einigen osteuropäischen Staaten, darunter Bulgarien (7,2 \%), Polen $(4,7 \%)$ und Slowenien (4,1 \%). In Slowenien dürfte die vergleichsweise hohe Nominal- lohnsteigerung mit der besonders starken Erhöhung des gesetzlichen Mindestlohns zusammenhängen, der $2010 \mathrm{um}$ mehr als $25 \%$ angehoben wurde (Schulten 2011a).

In Westeuropa hatte Großbritannien mit 3,2 \% die größte Nominallohnerhöhung, gefolgt von Dänemark und Schweden mit jeweils 2,7 \%. Deutschland lag mit 2,2 \% knapp über dem EU-Durchschnitt. Demgegenüber verzeichneten einige osteuropäische Länder sowie Griechenland und Irland einen teilweise kräftigen Rückgang der Nominallöhne. Letzteres dürfte vor allem an der Lohnentwicklung im öffentlichen Sektor liegen. Als Reaktion auf die Wirtschaftskrise und dem damit verbundenen Anstieg der öffentlichen Verschuldung sind zahlreiche europäische Regierungen dazu übergegangen, im öffentlichen Dienst Lohnkürzungen zu verordnen, die in Einzelfällen bis zu $30 \%$ ausmachten (Glassner 2010; Labour Research Department 2010).
Im Unterschied zu den Tarifabschlüssen hat die Effektivlohnentwicklung bereits 2009 mit deutlich geringeren $\mathrm{Zu}$ wachsraten stark auf die Krise reagiert. In vielen europäischen Ländern kam es demnach zu einer negativen Lohndrift, bei der die Effektivlohnerhöhungen deutlich hinter den Tariflohnzuwächsen zurückblieben. Eine zentrale Ursache hierfür lag in der Nutzung von Kurzarbeit und anderen Formen der Arbeitszeitverkürzung, die die Effektivlöhne reduziert haben. Im Jahr 2010 drehte sich in einigen europäischen Ländern wie Belgien, Frankreich und Deutschland mit dem Auslaufen der

\footnotetext{
4 Streng genommen werden in der AMECO-Datenbank der Europäischen Kommission keine "Löhne", sondern "Arbeitnehmerentgelte" (definiert als Bruttolöhne plus Arbeitgeberanteile zur Sozialversicherung) ausgewiesen. Der hier im Folgenden verwendete weite Lohnbegriff umfasst demnach die gesamten Arbeitskosten.
}

\section{Tabelle 3: Lohnentwicklung und Verteilungsbilanz in Europa 2008-2011*}

\begin{tabular}{|c|c|c|c|c|c|c|c|c|c|c|c|c|}
\hline & \multicolumn{4}{|c|}{ Nominallöhne } & \multicolumn{4}{|c|}{ Reallöhne } & \multicolumn{4}{|c|}{ Verteilungsbilanz } \\
\hline & 2008 & 2009 & 2010 & 2011 & 2008 & 2009 & 2010 & 2011 & 2008 & 2009 & 2010 & 2011 \\
\hline \multicolumn{13}{|l|}{ Alte EU-Staaten } \\
\hline Belgien & 3,6 & 1,8 & 1,1 & 3,1 & $-0,9$ & 1,8 & $-1,3$ & $-0,4$ & $-0,1$ & 4,2 & $-2,7$ & $-2,0$ \\
\hline Dänemark & 3,6 & 2,4 & 2,7 & 1,7 & 0,0 & 1,3 & 0,5 & $-0,8$ & 3,0 & 3,5 & $-3,7$ & $-2,4$ \\
\hline Deutschland & 2,0 & 0,2 & 2,2 & 2,7 & $-0,8$ & $-0,0$ & 1,0 & 0,1 & $-0,4$ & 4,7 & $-2,0$ & $-1,6$ \\
\hline Finnland & 5,1 & 1,7 & 2,0 & 2,8 & 1,2 & 0,1 & 0,3 & $-0,8$ & 1,8 & 5,7 & $-3,2$ & $-3,5$ \\
\hline Frankreich & 2,4 & 1,6 & 2,3 & 2,0 & $-0,8$ & 1,5 & 0,6 & $-0,3$ & $-0,3$ & 2,9 & $-0,9$ & $-1,2$ \\
\hline Griechenland & 7,0 & 3,6 & $-3,5$ & $-1,0$ & 2,8 & 2,2 & $-8,2$ & $-3,4$ & 2,0 & 3,6 & $-5,8$ & $-2,5$ \\
\hline Großbritannien & 1,5 & 2,5 & 3,2 & 2,8 & $-2,2$ & 0,4 & $-0,1$ & $-1,4$ & $-1,4$ & 3,7 & $-1,1$ & $-2,7$ \\
\hline Irland & 3,4 & 0,0 & $-1,9$ & $-0,3$ & 0,3 & 1,7 & $-0,3$ & $-1,3$ & 2,7 & 1,1 & $-3,5$ & $-3,5$ \\
\hline Italien & 3,8 & 1,5 & 2,0 & 1,5 & 0,3 & 0,8 & 0,4 & $-1,1$ & 1,2 & 3,4 & $-1,7$ & $-1,6$ \\
\hline Luxemburg & 2,1 & 1,8 & 1,6 & 2,0 & $-2,0$ & 1,8 & $-1,2$ & $-1,5$ & 1,2 & 6,4 & $-3,1$ & $-2,8$ \\
\hline Niederlande & 3,6 & 2,2 & 1,1 & 2,9 & 1,4 & 1,2 & 0,2 & 0,6 & 0,8 & 4,0 & $-2,2$ & $-0,8$ \\
\hline Österreich & 3,2 & 2,3 & 1,6 & 2,5 & 0,0 & 1,9 & $-0,1$ & $-0,3$ & $-0,5$ & 4,2 & $-1,1$ & $-1,9$ \\
\hline Portugal & 3,0 & 3,3 & 1,5 & $-0,3$ & 0,4 & 4,2 & 0,1 & $-3,6$ & 0,8 & 4,2 & $-2,8$ & $-2,9$ \\
\hline Schweden & 1,5 & 1,3 & 2,7 & 2,8 & $-1,8$ & $-0,7$ & 0,8 & 1,1 & $-0,3$ & 2,7 & $-3,6$ & $-1,0$ \\
\hline Spanien & 6,4 & 4,1 & 0,7 & 0,9 & 2,2 & 4,3 & $-1,3$ & $-2,1$ & 0,8 & 1,2 & $-3,6$ & $-3,5$ \\
\hline \multicolumn{13}{|l|}{ Neue EU-Staaten } \\
\hline Bulgarien & 16,3 & 9,4 & 7,2 & 7,1 & 4,4 & 7,0 & 4,2 & 2,8 & 0,9 & 9,9 & $-2,2$ & 0,5 \\
\hline Estland & 10,1 & $-3,3$ & $-0,2$ & 4,4 & $-0,5$ & $-3,5$ & $-3,0$ & $-0,3$ & 4,8 & 0,9 & $-11,3$ & $-0,9$ \\
\hline Lettland & 15,7 & $-12,2$ & $-6,5$ & 1,5 & 0,5 & $-15,4$ & $-5,2$ & $-1,9$ & 5,6 & $-9,9$ & $-9,9$ & $-3,7$ \\
\hline Litauen & 14,3 & $-11,1$ & $-1,3$ & 3,4 & 3,2 & $-15,3$ & $-2,5$ & 0,2 & $-0,4$ & $-6,8$ & $-9,3$ & $-2,6$ \\
\hline Malta & 4,9 & 2,9 & $-1,7$ & 2,0 & 0,2 & 1,1 & $-3,7$ & $-0,6$ & $-2,4$ & 4,1 & $-5,2$ & $-1,3$ \\
\hline Polen & 8,9 & 2,9 & 4,7 & 5,9 & 4,7 & $-1,1$ & 2,1 & 2,1 & 3,4 & $-2,4$ & $-1,3$ & $-0,8$ \\
\hline Rumänien & 31,9 & $-6,6$ & 1,3 & 2,2 & 24,0 & $-12,2$ & $-4,7$ & $-4,5$ & 16,7 & $-6,8$ & $-5,3$ & $-5,9$ \\
\hline Slowakei & 6,9 & 5,0 & 2,7 & 3,9 & 3,0 & 4,1 & 2,0 & 0,2 & 0,2 & 6,4 & $-3,5$ & $-2,7$ \\
\hline Slowenien & 7,0 & 1,6 & 4,1 & 2,4 & 1,4 & 0,8 & 2,0 & $-0,1$ & 0,5 & 7,1 & $-1,5$ & $-3,4$ \\
\hline Tschechien & 6,3 & 0,4 & 2,9 & 2,5 & 0,1 & $-0,2$ & 1,7 & 0,2 & $-1,1$ & 2,8 & $-1,4$ & $-1,8$ \\
\hline Ungarn & 7,0 & $-2,2$ & $-0,2$ & 2,6 & 1,0 & $-6,2$ & $-4,9$ & $-1,4$ & $-1,2$ & $-2,2$ & $-5,9$ & $-3,7$ \\
\hline Zypern & 2,3 & 3,2 & 2,8 & 3,8 & $-2,0$ & 3,1 & 0,3 & 0,4 & $-2,8$ & 4,1 & $-1,1$ & $-0,9$ \\
\hline EU 27 & 3,2 & 1,6 & 2,1 & 2,3 & $-0,5$ & 0,6 & 0,0 & $-0,8$ & $-0,7$ & 2,9 & $-2,2$ & $-2,2$ \\
\hline Norwegen & 6,2 & 3,6 & 3,8 & 3,5 & 2,7 & 1,3 & 1,5 & 1,6 & 5,0 & 2,1 & 0,8 & $-0,5$ \\
\hline Schweiz & 2,9 & 0,9 & $-1,1$ & 4,1 & 0,5 & 1,6 & $-1,8$ & 3,1 & 0,5 & 5,6 & $-2,1$ & 3,4 \\
\hline \multicolumn{13}{|c|}{$\begin{array}{l}\text { *Nominallöhne }=\text { Nominale Arbeitnehmerentgelte pro Arbeitnehmer, Veränderung in \% gegenüber dem Vorjahr. } \\
\text { Reallöhne = Nominale Arbeitnehmerentgelte pro Arbeitnehmer deflationiert um die Entwicklung des harmonisierten Verbrauchspreisindex, Veränderungen in \% gegenüber dem Vorjahr. } \\
\text { Verteilungsbilanz }=\text { Saldo des jährlichen Nominallohnzuwachses und des Verteilungsspielraums (vgl. Tabelle 2) in Prozentpunkten. } \\
\text { Angaben für 2011: Prognose der Europäischen Kommission. }\end{array}$} \\
\hline \multicolumn{9}{|c|}{ Ouelle: AMECO Datenbank der Europäischen Kommission, Version 13.5.2011; Berechnungen des WSI. } & & & \multicolumn{2}{|c|}{ MITTEILUNGEI } \\
\hline
\end{tabular}




\section{Abb. 4: Entwicklung der preislichen Wettbewerbsfähigkeit auf Basis der Lohnstückkostenentwicklung 1999-2010* - in \% -}

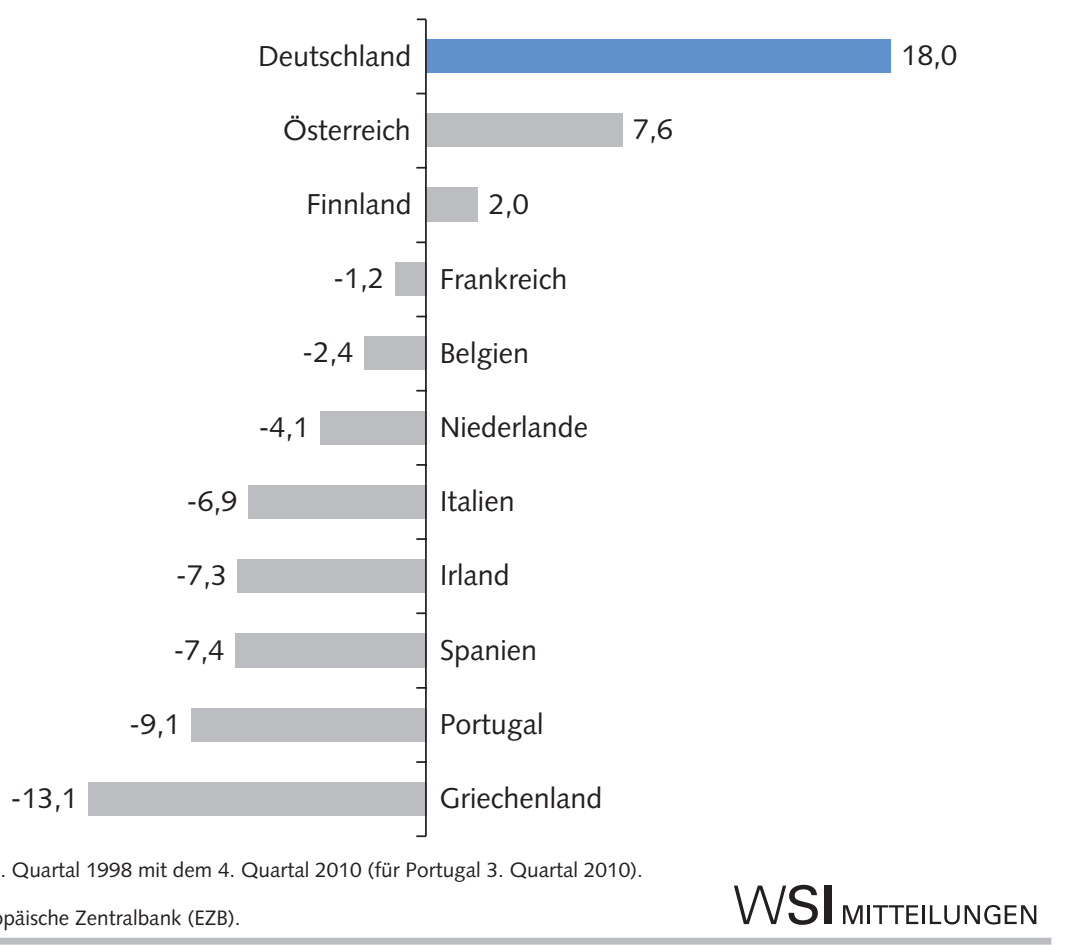

Quelle: Europäische Zentralbank (EZB).

päische Kommmission damit, dass es in diesem Jahr in 18 von 27 EU-Staaten zu einem Rückgang der Reallöhne kommen wird. EU-weit wird ein Reallohnrückgang von $0,8 \%$ prognostiziert, wobei die stärksten Reallohnverluste in Spanien, Portugal und Griechenland erwartet werden. Für Deutschland wird hingegen mit $0,1 \%$ ein minimaler Reallohnanstieg vorhergesagt.

Die Verteilungsbilanz, die sich aus dem Saldo aus Nominallohnentwicklung und dem Verteilungsspielraum ergibt, war 2010 in sämtlichen EU-Staaten negativ. Dies bedeutet, dass es in keinem EU-Land gelungen ist, den lohnpolitischen Verteilungsspielraum aus Preis- und Produktivitätsentwicklung auszuschöpfen. Im EU-Durchschnitt lag die Verteilungsbilanz 2,2 Prozentpunkte unterhalb des Verteilungsspielraums. Eine ähnlich negative Verteilungsbilanz wird von der Europäischen Kommission auch für 2011 erwartet. Nachdem krisenbedingt die Lohnentwicklung im Jahr 2009 kurzfristig über den (damals negativen) Verteilungsspielraum hinausschoss, ist sie bereits 2010 wieder auf einen restriktiven Kurs zurückgekehrt, der ein erneutes Absinken der Lohnquote und eine weitere Umverteilung zugunsten der Kapitaleinkommen nach sich zieht.

\section{Ausblick: Anhaltend schwache Lohnentwick- lung in Europa}

Die Lohnentwicklung in Europa nimmt insgesamt derzeit einen äußerst schwachen Verlauf. Während 2010 EU-weit die Reallöhne stagnieren, kommt es 2011 aller Voraussicht nach sogar zu einem deutlichen Reallohnrückgang. Besonders betroffen hiervon sind die sogenannten PIGS-Staaten (Portugal, Irland, Griechenland und Spanien), die derzeit im Zentrum der europapolitischen Debatte stehen und von EU-Seite als Hauptschuldige der aktuellen „Euro-Krise“ ausgemacht werden. Eine ähnliche Entwicklung findet sich aber auch nach wie vor in zahlreichen osteuropäischen Staaten. Unter massivem Druck des Internationalen Währungsfonds (IWF) und der EU sind all diese Staaten dazu übergegangen, die Löhne im öffentlichen Sektor drastisch zu kürzen und damit zugleich das Signal für eine restriktive Lohnentwicklung in der Privatwirtschaft vorzugeben.

In denjenigen Ländern, in denen es insbesondere durch einen erneuten Aufschwung der Exportwirtschaft gelungen ist, wieder aus der Krise herauszukommen, fällt die Lohnentwicklung zwar durchschnittlich etwas stärker aus. Aber auch diese Länder bleiben deutlich hinter ihren Möglichkeiten und weisen ebenfalls eine negative Verteilungsbilanz aus. Deutschland konnte seine langjährige lohnpolitische Schlusslichtposition zwar einstweilen abgeben. Dies lag jedoch weniger an einer besonders expansiven Lohnpolitik, als vielmehr daran, dass in den meisten übrigen EU-Staaten die gesamtwirtschaftliche Lohnentwicklung noch schwächer ausfiel.

Von der Lohnentwicklung gehen damit derzeit kaum positive Impulse aus, um die strukturellen ökonomischen Probleme in Europa zu überwinden und eine nachhaltige Wachstumsstrategie einzuleiten. In den Krisenländern führt der anhaltende Reallohnverlust im Gegenteil dazu, dass die gesamtwirtschaftliche Nachfrage immer weiter zurückgeht und sich die Krise weiter zuspitzt. In den exportorientierten Überschussländern ist die Lohnentwicklung hingegen immer noch zu schwach, um eine deutlichere Stärkung der Binnen- 
konjunktur zu befördern und damit einen Beitrag zu leisten, die Ungleichgewichte in Europa abzubauen.

Seit der Einführung der Europäischen Währungsunion hat die unterschiedliche Entwicklung der Lohnstückkosten wesentlich dazu beigetragen, dass sich die preisliche Wettbewerbsfähigkeit einiger weniger Staaten immer weiter verbesserte, während sie in den meisten anderen Staaten immer schlechter wurde (Abbildung 4). Allen voran hat Deutschland mit Hilfe seiner lohnpolitischen Sonderrolle sein exportgetriebenes Wachstumsmodell immer weiter ausbauen können.

Auf europäischer Bühne ist der deutsche Entwicklungsweg kurzzeitig von einigen prominenten Akteuren wie der französischen Finanzministerin Christine Lagarde oder dem Luxemburgischen Ministerpräsidenten Jean-Claude Juncker offen als „Beggar-my-Neighbour-Politik“ und nationales Lohndumping kritisiert worden (Schulten 2011b). Die bis dato relativ erfolgreiche Überwindung der Wirtschaftskrise in Deutschland hat diese Kritik jedoch weitgehend verstummen lassen. An ihre Stelle ist nunmehr das Lob für den deutschen Entwicklungsweg getreten. So hat z.B. der Präsident der EZB, JeanClaude Trichet, die restriktive Lohnpolitik in Deutschland und die hierbei zugrunde liegenden Reformen auf dem Arbeitsmarkt offen als „Vorbild“ für Europa propagiert (Trichet 2010). Mit der Verabschiedung des sogenannten „Euro-Plus-Pakt“ im März 2011 hat das „deutsche Vorbild“ schließlich auch offiziell Eingang in die EU-Politik gefunden (Rat der Europäischen Union 2011). Erstmals wird hier vonseiten der EU explizit ein Ansatz für eine europäische Koordinierung der Lohnpolitik formuliert, bei dem möglichst alle EU-Staaten dem deutschen Weg folgen und durch eine restriktive Lohnentwicklung ihre Wettbewerbsfähigkeit verbessern sollen.

Die Verallgemeinerung des deutschen Entwicklungsweges wird jedoch endgültig dazu führen, dass europaweit eine negative Lohnsenkungsspirale in Gang gesetzt wird, in deren Folge sich die strukturellen ökonomischen Probleme in Europa weiter verschärfen werden. Die Europäischen Gewerkschaften weisen demgegenüber in ihrer Kritik am Euro-Plus-Pakt darauf hin, dass die Lohnpolitik in der nationalen
Autonomie der Tarifvertragsparteien liegt und die EU keinerlei vertragliche Kompetenzen hat, in diese zu intervenieren (EGB 2011). Eine reine Verteidigung der nationalen Lohnpolitiken greift jedoch zu kurz. So haben die europäischen Gewerkschaften selbst im letzten Jahrzehnt eine Reihe von Ansatzpunkten für eine europäische Koordinierung der Lohnpolitik entwickelt, die eine grundlegende Alternative zum vorherrschenden Kurs der EU markieren (Schulten 2004). Aus heutiger Sicht geht es dabei im Kern vor allem um zwei Dinge (Busch/Hirschel 2011): Zum einen müssen in den Krisenländern weitere Lohnkürzungen verhindert und es muss zu einer produktivitätsorientierten Reallohnpolitik zurückgefunden werden, um die binnenwirtschaftliche Stagnation zu überwinden. Zum anderen stehen die Überschussländer - darunter allen voran Deutschland - vor der Aufgabe, durch eine deutlich expansivere Lohnentwicklung die Fehlentwicklungen des letzten Jahrzehntes zumindest ein Stück weit zu korrigieren und ihre Verantwortung für die wirtschaftliche Dynamik in ganz Europa wahrzunehmen.

\section{LITERATUR}

Busch, K./Hirschel, D. (2011): Europa am Scheideweg. Wege aus der Krise, hrsg. von der Friedrich-Ebert-Stiftung, Reihe Internationale Politikanalyse, März, Bonn

Carley, M. (2010): Pay Developments 2009, European Industrial Relations Observatory (EIRO), http://www.eurofound.europa.eu/eiro/ studies/tn1004029s/tn1004029s.htm

Europäischer Gewerkschaftsbund (EGB) (2011): Das Athener Manifest, beschlossen auf dem EGB Kongress vom 16.-19. Mai in Athen

Europäische Kommission (2011): European Economic Forecast - Spring 2011, European Economy (1), http://ec.europa.eu/economy_finance/ publications/european_economy/2011/pdf/ee-2011-1_en.pdf

Glassner, V. (2010): The Public Sector in the Crisis, ETUI Working Paper 2010.07, Brüssel

Institut für Makroökonomie und Konjunkturforschung (IMK)/ L'Observatoire Français des Conjonctures Économiques (OFCE)/ Österreichisches Institut für Wirtschaftsforschung (WIFO) (2011): Der Euroraum im Umbruch. Erste gemeinsame Diagnose des MakroKonsortiums, IMK Report (61), April, Düsseldorf
Labour Research Department (2010): The wrong Target - how Governments are making Public Sector Workers pay for the Crisis, Studie im Auftrag des Europäischen Gewerkschaftsverbandes für den Öffentlichen Dienst (EGÖD), Brüssel, http://www.epsu.org/IMG/pdf/ Pay_cuts_report.pdf

Rat der Europäischen Union (2011): Der Euro-Plus-Pakt. Stärkere Koordinierung der Wirtschaftspolitik im Hinblick auf Wettbewerbsfähigkeit und Konvergenz, Schlussfolgerungen des Europäischen Rats vom 24./25. März

Schulten, T. (2004): Solidarische Lohnpolitik in Europa, Hamburg Schulten, T. (2010): Das deutsche Tarifvertragssystem im europäischen Vergleich, in: Bispinck, R./Schulten, T. (Hrsg.): Zukunft der Tarifautonomie, Hamburg, S. 193-204

Schulten, T. (2011a): WSI-Mindestlohnbericht 2011 - Unterschiedliche Strategien in der Krise, in: WSI-Mitteilungen 64 (3), S. 152-160 Schulten, T. (2011b): Deutschlands lohnpolitische Sonderrolle in Europa, in: Bispinck, R. (Hrsg.): Zwischen Beschäftigungswunder und Lohndumping? Tarifpolitik in und nach der Krise, Hamburg, S. 47-58 Trichet, J.-C. (2010): Interview mit Jean-Claude Trichet, in: Le Figaro vom 3. September 